\title{
Controle instrucional e relaxamento como preparo psicológico pré-cirúrgico para portadores de cardiopatia ${ }^{1}$
}

\author{
Instructional control and relaxation procedure as \\ psychological preparation for pre-surgery \\ patients with heart disease
}

Maria Estela Martins SILVA²

Norma Sant'Ana ZAKIR ${ }^{3}$

\begin{abstract}
Resumo
Avaliou-se um preparo pré-cirúrgico baseado em instrução e relaxamento na evolução clínica e comportamento de pacientes submetidos à cirurgia cardíaca em hospital geral no interior do Paraná. Vinte pacientes com idade entre 51 e 73 anos, com condição socioeconômica de baixa a média-alta, foram distribuídos aleatoriamente em grupo experimental e controle. O grupo experimental participou de uma sessão de instrução e outra de relaxamento antes da cirurgia e o grupo-controle recebeu o preparo padrão realizado pelo hospital. Os efeitos da intervenção foram avaliados por nível de cortisol sanguíneo, Inventário de Ansiedade de Beck, comportamento na Unidade de Terapia Intensiva e evolução clínica. Foram encontradas diferenças estatísticas significantes entre o grupo experimental e o grupo-controle em relação ao comportamento menos agitado na Unidade de Terapia Intensiva, nível de cortisol sanguíneo antes da cirurgia, decréscimo no grau de ansiedade intragrupo e evolução clínica. Conclui-se que a intervenção parece ter produzido efeito positivo no comportamento e na evolução clínica do paciente.
\end{abstract}

Unitermos: Controle instrucional. Preparo psicológico pré-cirúrgico. Psicologia da saúde. Relaxamento.

\begin{abstract}
The aim of this study was to evaluate the effect of preoperative preparation based on instruction and relaxation in the clinical evolution and the behavior of patients undergoing cardiac surgery, in a general hospital in the state of Paraná. Twenty patients aged between 51 and 73 , with a socio-economic status between low and medium-high, were randomly divided into experimental and control groups. The experimental group participated in one session of instruction and another of relaxation before surgery and the control group received standard preparation performed by the hospital. Intervention effects were evaluated by blood cortisol level, Beck Anxiety Inventory, patient behavior in the Intensive Care Unit and the clinical outcome. We found statistically significant differences between the experimental group and the control group in terms of less agitated behavior in the Intensive Care Unit, blood cortisol level before surgery, a decrease in the degree of anxiety and intra-group clinical outcomes. The intervention appears to have produced positive effects on behavior and clinical outcomes.
\end{abstract}

Uniterms: Instructional control. Preoperative psychological preparation. Health psychology. Relaxation.

\section{$\boldsymbol{\nabla \nabla \nabla \nabla}$}

1 Artigo elaborado a partir da dissertação de M.E.M. SILVA, intitulada "Efeitos de uma intervenção breve em preparo psicológico pré-cirúrgico na evolução clínica de portadores de cardiopatia". Universidade Estadual de Londrina, 2007.

2 Núcleo de Educação Continuada do Paraná. Maringá, PR, Brasil.

3 Universidade Estadual de Londrina, Curso de Psicologia, Departamento de Psicologia Geral e Análise do Comportamento. Rod. Celso Garcia Cid (PR 445), Km 380, 86051-990, Londrina, PR, Brasil. Correspondência para/Correspondence to: N.S. ZAKIR. E-mail: <nszakir@uel.br>. 
A compreensão do impacto da cirurgia cardíaca requer análise ampla das circunstâncias críticas da situação que extrapolam o ato médico em si. Esta análise deve orientar-se, em parte, pelos fundamentos de uma das recentes especialidades contemporâneas: a psicologia da saúde.

Reconhecida oficialmente pela American Psychological Association em 1997, a psicologia da saúde afirmou-se com o objetivo geral de intervir na prevenção e na reabilitação de doenças, e de auxiliar no manejo e na diminuição do impacto de procedimentos médicos invasivos sobre o paciente para reduzir reações emocionais de medo, ansiedade e dor, inclusive em casos de procedimento cirúrgico e promoção da adesão ao tratamento (Costa Jr., 2001; Costa Jr., 2005; Kain et al., 2007; Miyazaki, Domingos \& Caballo, 2001). A adesão, medida pelo grau com que o indivíduo aceita ou coopera com o tratamento (Galimbert et al., 2007), em uma perpectiva analítico-comportamental, define-se pelo grau de controle sobre uma classe de operantes entre os quais se incluem respostas chamadas de auto-cuidado (Malerbi, 2001).

À semelhança de psicólogos de outras abordagens, o analista do comportamento atua na área da saúde e compõe equipes multidisciplinares para atuar no planejamento e na execução de procedimentos duradouros ou intervenções breves (Amaral, 2001). As intervenções breves são denominadas focais, pois se orientam para problemas delimitados ao contexto em que ocorrem, e"envolvem análises contingenciais no aqui e agora" (Amaral, 2001, p.280). São úteis no contexto da saúde, visto que o tempo é uma variável relevante no processo de tratamento, em especial para o paciente submetido à intervenção cirúrgica.

O paciente submetido à cirurgia cardíaca pode estar sujeito a contingências mais aversivas do que aqueles em tratamento clínico, pois, além de todas as variáveis implícitas na doença e na hospitalização, o paciente está suscetível a sensações de dor, a infecções, a intervenções invasivas e à ameaça de algo muito arriscado (Gorayeb, 2001; Santana, Fernandes, Zanin, Waeteman \& Soares, 2010). A possibilidade de ocorrer complicações pós-operatórias é grande, pois há risco de infecção, infarte, hemorragia, insuficiência hepática e desalinhamento do osso esterno.

Riscos e desconfortos podem ser potencializados

372 ou minimizados em função do estresse e do repertório de enfrentamento do paciente. Um comportamento agitado pode afetar a evolução clínica, enquanto a adesão e o relaxamento incluem comportamentos funcionais em termos de sobrevivência no ambiente hospitalar (Garbossa, Maldaner, Mortari, Biasi \& Leguisamo, 2009; Pires, 2001; Santana et al., 2010). A agitação, porém, é provável de ocorrer em um contexto altamente estressor para a maioria dos indivíduos, como o ambiente hospitalar (Souza \& Souza Filho, 2008). A alta probabilidade de comportamento agitado e as implicações de sua ocorrência são indicadores da relevância do preparo psicológico pré-cirúrgico, em geral, e em cirurgias cardíacas, em particular.

Uma das formas de alterar a função aversiva de eventos ambientais é o uso de instruções pré-cirúrgicas que podem prevenir as respostas de agitação mais prováveis, porém, indesejáveis, e favorecer formas mais adaptativas de reagir à situação (Shimidt \& Souza, 2003; Valgas, 2004). O controle que essa operação de estímulos (apresentação de instruções) exerce no comportamento é chamado de controle instrucional e baseia-se no fato da instrução alterar a função de outros estímulos presentes no ambiente (Valgas, 2004).

Ao seguir instruções, uma pessoa não precisa se submeter às contingências naturais e possivelmente ao risco de situações específicas que implicam dano grave em caso de ausência de efetividade do comportamento (Catania, 1999; Haydu, 2009; Schmidt \& Souza, 2003). Para que a instrução ao paciente seja eficaz, ela deve incluir explicações sobre os procedimentos e descrever o que o paciente pode vir a sentir, aumentando a previsibilidade dos eventos, e especificar como ele pode colaborar (Amaral \& Albuquerque, 2000).

A instrução pode agir preventivamente também com relação à dor e ao desconforto. Um paciente ligado a um respirador mecânico provavelmente tentaria desvencilhar-se dele devido ao desconforto que provoca, mas poderá aquiescer ao ritmo de respiração da máquina e relaxar a musculatura se for instruído para tal. A resposta de relaxar poderá ser produzida ao invés da resposta de agitar-se, e o desconforto será diminuído, reduzindo a aversão à situação e fortalecendo o operante para seguir a instrução.

Outro recurso bastante utilizado pela abordagem cognitivo-comportamental que pode funcionar como fonte de reforçamento positivo é o treino de relaxamento 
que consiste na modelagem de operantes que são acompanhados da diminuição generalizada da ação do sistema nervoso simpático e da atividade metabólica (Silva Jr., 2000). Técnicas de relaxamento levam à diminuição da frequência, intensidade e duração da dor e ao aumento do fluxo sanguíneo cerebral, elevando a produção pelo hipotálamo de neurotransmissores como endorfina e serotonina, substâncias que apresentam uma estrutura química similar à da morfina e que, por causa dessa semelhança, apresentam um efeito analgésico (Smith, 1996).

Muitos trabalhos que têm sido realizados na área da enfermagem, da fisioterapia e da psicologia avaliam o papel do preparo pré-cirúrgico na redução da ansiedade do paciente cardiopata e na melhora da evolução clínica (Garbossa et al., 2009; Kain et al., 2007; Marcolino, Suzuki, Alli, Gozzani \& Mathias, 2007; Santana et al., 2010; Umann, Guido \& Linch, 2010; Werlang, Azzolin, Moraes \& Souza, 2008). A revisão de literatura realizada por Broering e Crepaldi (2008) mostra que a maioria dos programas de preparo psicológico para crianças submetidas a condutas médicas utilizou tratamento comportamental cognitivo, por terem sido os mais estudados e os mais testados.

Apesar de muitas pesquisas apontarem para a efetividade do preparo pré-cirúrgico, outras pesquisas mostram que o uso de intervenções breves encontram limitações em sua efetividade que dizem respeito à gravidade e à complexidade da situação e às limitações de recursos, inclusive temporais. A transmissão de informações não é o suficiente para modificar comportamentos de forma duradoura, sendo necessário desenvolver e fortalecer categorias específicas de resposta e estratégias mais adequadas de enfrentamento (Santana et al., 2010). Há, ainda, a variabilidade entre os pacientes, pois cada um apresenta uma história com relação às interações entre stress e enfretamento de situações estressoras, delineadas desde muito cedo no ciclo vital (Lipp, Frare \& Santos, 2007; Zakir, 2010).

Diante das pesquisas apresentadas, que apontam para a importância e para as limitações desse tipo de intervenção, foi objetivo deste estudo verificar o efeito de um preparo psicológico pré-cirúrgico breve - baseado em instrução e relaxamento -, no comportamento ao despertar da anestesia, no grau de ansiedade e de stress, e na evolução clínica de pacientes submetidos à cirurgia cardíaca.

\section{Método}

\section{Participantes}

Participaram do estudo 20 pessoas de ambos os sexos, portadoras de doença cardíaca com indicação para cirurgia de revascularização do miocárdio e/ou troca valvar, com idade entre 51 e 73 anos, de nível socioeconômico de baixo a médio-alto. Todos os participantes da pesquisa foram operados pelo mesmo cirurgião, no mesmo hospital geral de porte médio do noroeste do Paraná, que atende pacientes da rede pública, particular e convênios. Os critérios de seleção foram: ter idade inferior a 80 anos e não apresentar comorbidade associada com insuficiência renal ou outras doenças vasculares. Também não participaram da pesquisa pessoas internadas e submetidas à cirurgia em caráter emergencial ou que estavam em tratamento na Unidade de Terapia Intensiva (UTI) antes da cirurgia. Essas restrições foram estabelecidas com base nas informações acerca da maior morbidade e mortalidade envolvidas nessas situações.

Os participantes foram distribuídos aleatoriamente em dois grupos, Grupo Experimental (GE) e Grupo-Controle (GC), obedecendo a ordem de internação no hospital (Tabela 1).

A maioria dos participantes não teve nenhum contato anterior com psicólogo e relaxamento. Dois participantes do grupo experimental já tinham se consultado com um psicólogo, mas não tiveram contato com técnicas de relaxamento.

\section{Instrumentos}

Inventário de Ansiedade de Beck (BAl) (Beck, Brown, Epstein \& Steer, 1988): o instrumento foi traduzido, adaptado, validado e normatizado no Brasil por Cunha (1999), e a correção dos resultados foi feita utilizando-se os valores normativos brasileiros.

Exame laboratorial do nível de cortisol sanguíneo: esse exame foi incluído como medida objetiva de estresse dos participantes. Os resultados dos grupos GC e GE foram analisados estatisticamente pelo valor absoluto do exame, e qualitativamente de acordo com os critérios de normalidade: estresse semelhante à média da população (valores entre 5,0 a 25ng/dL para coleta pela manhã e 2,5 a 12,5ng/dL para coleta à tarde). 
Tabela 1. Dados gerais dos participantes da pesquisa. Maringá (PR), 2006.

\begin{tabular}{|c|c|c|c|c|}
\hline \multirow{2}{*}{ Características } & & \multicolumn{3}{|c|}{ Sujeitos (n) } \\
\hline & & $\mathrm{GE}(\mathrm{n}=10)$ & $\mathrm{GC}(\mathrm{n}=10)$ & Total $(n=20)$ \\
\hline \multirow[t]{2}{*}{ Sexo } & Feminino & 6 & 3 & 9 \\
\hline & Masculino & 4 & 7 & 11 \\
\hline Total & & 10 & 10 & 20 \\
\hline \multirow[t]{3}{*}{ Idade } & 50 a 59 anos & 5 & 4 & 9 \\
\hline & 60 a 69 anos & 4 & 5 & 9 \\
\hline & 70 anos ou mais & 1 & 1 & 2 \\
\hline Total & & 10 & 10 & 20 \\
\hline \multirow[t]{4}{*}{ Escolaridade } & Fundamental incompleto & 6 & 8 & 14 \\
\hline & Fundamental complleto & 1 & 1 & 2 \\
\hline & Ensino médio & 3 & 0 & 3 \\
\hline & Superior & 0 & 1 & 1 \\
\hline Total & & 10 & 10 & 20 \\
\hline \multirow[t]{2}{*}{ Religião } & Católica & 7 & 9 & 16 \\
\hline & Evangélica & 3 & 1 & 4 \\
\hline Total & & 10 & 10 & 20 \\
\hline \multirow[t]{2}{*}{ Tipo de cirurgia cardíaca } & Revascularização do miocárdio & 6 & 7 & 13 \\
\hline & Troca Valvar & 4 & 3 & 7 \\
\hline Total & & 10 & 10 & 20 \\
\hline \multirow[t]{4}{*}{ Há quanto tempo sabe da necessidade de cirurgia } & Menos de 1 semana & 2 & 2 & 4 \\
\hline & Entre 1 semana e 1 mês & 0 & 3 & 3 \\
\hline & Entre 1 a 6 meses & 5 & 4 & 9 \\
\hline & Mais de 6 meses & 3 & 1 & 4 \\
\hline Total & & 10 & 10 & 20 \\
\hline \multirow{2}{*}{$\begin{array}{l}\text { Contato anterior com tratamento psicológico e } \\
\text { relaxamento }\end{array}$} & Sim & 2 & 0 & 2 \\
\hline & Não & 8 & 10 & 18 \\
\hline Total & & 10 & 10 & 20 \\
\hline \multirow[t]{2}{*}{ Outros estressores além da cirurgia } & Sim & 7 & 3 & 10 \\
\hline & Não & 3 & 7 & 10 \\
\hline Total & & 10 & 10 & 20 \\
\hline
\end{tabular}

GC: grupo-controle; GE grupo-experimental.

Formulário do Profissional de Saúde - Comportamento adaptativo/desadaptativo na UTI: alguns comportamentos frequentes no ambiente da UTI foram classificados pela equipe de saúde (psicóloga, médico e enfermeira) como adaptativos ou desadaptativos, e um formulário para registro da frequência desses comportamentos foi elaborado por essa mesma equipe. Para que fosse realizada uma análise quantitativa dos resultados, foi atribuída uma nota para cada alternativa na escala utilizada no formulário (Tabela 2).

Ficha de acompanhamento da evolução clínica: o prontuário do paciente é o documento no qual a equipe de saúde (médicos, enfermeiros e técnicos de

374 enfermagem) registra as medidas aferidas a respeito da condição clínica do paciente a cada 6 horas. Os dados coletados no prontuário do paciente foram registrados na Ficha de Acompanhamento da Evolução Clínica, desenvolvida especialmente para o estudo pelas autoras. A cada variável da evolução clínica foram atribuídas notas para que uma análise quantitativa fosse possível (Tabela 3).

\section{Procedimentos}

Para realização do estudo, o projeto foi submetido à avaliação, sendo aprovado pelo comitê de ética em pesquisa do hospital onde se realizou a intervenção e pelo comitê de ética em pesquisa envolvendo seres 
Tabela 2. Atribuição de nota do formulário do profissional de saúde. Maringá (PR), 2006.

\begin{tabular}{llc}
\hline Questão & Alternativas & Valor \\
\hline 1) Dia e hora do despertar & Questão aberta & Nulo \\
2) Durante o despertar, o paciente apresentou... & Agitação intensa & 0 \\
& Agitação moderada & 1,0 \\
& Movimentos brandos & 2,0 \\
3) Logo ao despertar... & Pouco/nenhum movimento & 3,0 \\
& Olhos muito abertos & 0 \\
& Busca ativa & 1,0 \\
4) Adaptação à ventilação mecânica & Abriu os olhos às vezes & $3,0^{*}$ \\
& Olhos fechados & 2,0 \\
& Ruídos e agitação & 0 \\
& Ruídos mas acalmou-se & 1,0 \\
5) Número de solicitações à enfermagem & Ruídos sem agitação & 2,0 \\
& Sem ruídos & 3,0 \\
& Solicitação muito frequente & 0 \\
6) Tipo de solicitação & Solicitação normal & 1,0 \\
7) Aspecto emocional geral & Pouca solicitação & 2,0 \\
& Nenhuma solicitação & 3,0 \\
& Questão aberta & Nulo \\
& Muito ansioso & 0 \\
& Pouco ansioso & 1,0 \\
& Calmo & 2,0 \\
& Muito calmo & 3,0 \\
\hline
\end{tabular}

*A pontuação não segue a ordem regular, pois a terceira alternativa é mais desejável do que a quarta alternativa no que se refere a um comportamento adequado.

Tabela 3. Critério para atribuição de nota da evolução clínica. Maringá (PR), 2006.

\begin{tabular}{llc}
\hline Variável & Critério & Nota \\
\hline Pressão arterial & $100 / 60^{*} \mathrm{mmHg}<\mathrm{PA}<150 / 90 \mathrm{mmHg}$ & 2,0 \\
& $100 / 60 \mathrm{mmHg}>\mathrm{PA}>150 / 90 \mathrm{mmHg}$ & 0 \\
Temperatura & $36,0^{\circ} \mathrm{C}<\mathrm{t}<37,2^{\circ} \mathrm{C}$ & 2,0 \\
& $36,0^{\circ} \mathrm{C}>\mathrm{t}>37,2^{\circ} \mathrm{C}$ & 0 \\
Taxa cardíaca & $60 \mathrm{bpm}<\mathrm{TC}<80 \mathrm{bpm} 60 \mathrm{bpm}$ & 2,0 \\
& $>$ TC>80bpm & 0 \\
Orientação espaço-temporal & Orientado & 1,0 \\
& Desorientado & 0 \\
& Comunicativo & 1,0 \\
Comunicação & Pouco ou não comunicativo & 0 \\
Deambulação & Deambulando & 1,0 \\
Calma/agitação & Repouso relativo/repouso & 0 \\
Aceitação da dieta & Calmo & 1,0 \\
& Agitado & 0 \\
\hline
\end{tabular}

"O valor da pressão arterial se compõe de dois valores em miligramas de mercúrio: a medida da pressão sistólica e diastólica PA= (PS/PD). mmHg: milímetros de mercúrio; PA: pressão arterial; t: temperatura; ${ }^{\circ} \mathrm{C}$ : graus Celsius; TC: taxa cardíaca; bpm: batimentos por minuto. 
humanos da Universidade Estadual de Londrina (CEP- Protocolo n 012/06 em 17/05/2006). Durante o contato inicial com os participantes, foi assinado o Termo de Consentimento Livre e Esclarecido, aplicou-se o Inventário de Ansiedade de Beck (Beck, Brown, Epstein \& Steer, 1988) e foi realizada a coleta de dados pessoais.

A partir desse ponto, os pacientes do grupo-controle receberam o preparo pré-operatório padrão realizado pela equipe de saúde do hospital (médico, enfermeiros e fisioterapeuta), que consiste de informações sobre o tratamento e a cirurgia, de forma assistemática. O grupo experimental foi submetido a uma sessão de 40 minutos de treino em relaxamento e sessão de 20 minutos com instruções sobre o comportamento adequado ao despertar na UTI, além do preparo padrão do hospital.

Todos os participantes tiveram amostra de sangue colhida antes da cirurgia para realização de exame laboratorial de cortisol sanguíneo. A coleta foi realizada pela manhã ou à tarde de acordo com o horário em que seria realizada a cirurgia.

A enfermeira chefe da UTI cardíaca observou os pacientes durante o despertar da anestesia e avaliou seu comportamento como adaptativo ou desadaptativo, utilizando o Formulário do Profissional de Saúde. A mesma enfermeira fez a observação do comportamento de todos os participantes, sem ter conhecimento do grupo a que o paciente pertencia: controle ou experimental.

Durante a internação foi registrada a evolução clínica dos pacientes participantes da pesquisa na Ficha de Acompanhamento da Evolução Clínica, e todos os participantes responderam ainda ao Inventário BAI (Beck et al., 1988) pela segunda vez após a alta da UTI.

\section{Resultados e Discussão}

Os efeitos da intervenção foram avaliados quantitativamente e qualitativamente pelo registro e tratamento de várias medidas, intergrupo e intragrupo, com análise estatística utilizando os testes $t$ de Student e $U$ de Mann-Whitney, com nível de significância de $\alpha=0,05$.

Os resultados da aplicação do Inventário BAI (Beck et al., 1988) aos dois grupos - GE e GC - antes e 376 após a cirurgia não mostraram diferenças significativas entre as médias dos escores de ansiedade na situação de pré-teste nem de pós-teste entre os grupos. A análise intragrupo realizada pelo teste $U$ de Mann-Whitney indicou diferença estatisticamente significativa entre a situação de pré-teste e pós-teste com relação ao grupo experimental, com p (bilateral) =0,0323. Essa análise aponta para o fato de os participantes do grupo experimental aparentemente apresentarem diminuição no escore de ansiedade de forma mais acentuada do que os participantes do grupo-controle, comparando-se os valores de pré-teste e pós-teste.

O tratamento e a análise dos dados obtidos mediante o registro do comportamento do paciente ao acordar na UTI indicaram que a média das notas do grupo-controle foi igual a 9,6 e a média do grupo experimental foi de 12,9. A análise estatística desses resultados pelo teste $t$ de Student, com $p=0,003$, mostrou que há diferença significativa no desempenho dos grupos experimental e controle, o que sugere ter sido a intervenção efetiva para desenvolver no paciente um comportamento mais adaptativo ao despertar da anestesia.

Esses resultados podem ter sido obtidos por meio de três operações de estímulos concomitantes possivelmente presentes na intervenção. Analisando-se os dados do ponto de vista de relações que envolvem o comportamento verbal (instruções) e as emoções, é possível supor a ocorrência de transformações na função de estímulos (Shimidt \& Souza, 2003; Valgas, 2004). Em segundo lugar, é possível que o treino em relaxamento tenha funcionado como uma variável concorrente à agitação, já que geralmente o treino induz uma resposta incompatível com a tensão e os movimentos bruscos (Costa Jr., 2001). A terceira operação pode ter se dado pela introdução da imaginação dirigida, que pode ter propiciado distração da estimulação aversiva, e reforçamento positivo pelo caráter apetitivo da imagem sugerida.

A análise estatística dos resultados do exame de cortisol com teste $t$ de Student encontrou diferenças significativas $(p=0,031)$ entre as médias dos grupos GC e GE, indicando que a intervenção pode ter influenciado o grupo experimental, que apresentou grau de stress menor que o grupo-controle (Tabela 4). Visto que não foi aplicado qualquer inventário de sintomas de stress, nenhum dado foi coletado acerca do tipo de stress, se 
agudo ou crônico, e da fase do stress que os pacientes enfrentavam.

Quanto ao registro da evolução clínica dos participantes, os resultados foram analisados com referência à média geral dos grupos experimental e controle, e em cada período de internação (pré-operatório, UTI e pósoperatório). A análise estatística desses dados com teste $t$ de Student e $p=0,017$ demonstrou diferença nas médias da evolução clínica dos grupos experimental $(9,15)$ e controle $(8,4)$, com resultado significativamente superior para o grupo experimental.

Visto que o alto nível de stress e de ansiedade produz a diminuição da imunocompetência (Manfro et al., 2003), e sendo esta um fator bastante relevante para a recuperação do paciente, como apontou o estudo de Garbossa et al. (2009), é possível que o menor nível de stress tenha contribuído para que o grupo experimental apresentasse menos infecções, mantendo a temperatura

Tabela 4. Resultado do exame laboratorial de cortisol. Maringá (PR), 2006.

\begin{tabular}{|c|c|c|c|c|c|}
\hline \multirow{3}{*}{ Participante } & \multirow{2}{*}{\multicolumn{2}{|c|}{$\begin{array}{l}\text { Grupo-Controle } \\
\text { Cortisol (ng/dL) }\end{array}$}} & \multicolumn{3}{|c|}{ Grupo-Experimental } \\
\hline & & & \multirow{2}{*}{ Participante } & \multicolumn{2}{|c|}{ Cortisol (ng/dL) } \\
\hline & Manhã & Tarde & & Manhã & Tarde \\
\hline 1 & 11,9 & - & 2 & 8,6 & - \\
\hline 3 & 5,6 & - & 4 & - & 11,3 \\
\hline 5 & 10,3 & - & 6 & - & 3,2 \\
\hline 7 & - & 8,9 & 8 & - & 9,7 \\
\hline 9 & 18,5 & - & 10 & - & 6,2 \\
\hline 11 & 9,7 & - & 12 & - & 10,9 \\
\hline 13 & 36,2 & - & 14 & - & 5,7 \\
\hline 15 & - & 13,2 & 16 & - & 12,1 \\
\hline 17 & - & 9,4 & 18 & 2,9 & - \\
\hline 19 & - & 20,5 & 20 & - & 13,4 \\
\hline Média & 15,36 & 13,0 & Média & 5,75 & 9,06 \\
\hline
\end{tabular}

$\mathrm{ng} / \mathrm{dL}$ : nanograma de cortisol por decilitro de sangue.

Valores de referência: manhã - 5,0 a $25 \mathrm{ng} / \mathrm{dL}$ e tarde - 2,5 a 12,5ng/dL.

Tabela 5. Resultados da pesquisa e análise estatística. Maringá (PR), 2006.

\begin{tabular}{|c|c|c|c|c|c|c|c|c|c|}
\hline \multirow{3}{*}{$\begin{array}{l}\text { Instrumento } \\
\text { Inventário de } \\
\text { Ansiedade de }\end{array}$} & \multicolumn{4}{|c|}{ Grupo Experimental } & \multicolumn{4}{|c|}{ Grupo-Controle } & \multirow{3}{*}{$\begin{array}{c}\text { Análise Estatística } \\
\alpha=0,05^{*} \text { estatisticamente significante } \\
\text { Intergrupo - teste } t \text { de Student }\end{array}$} \\
\hline & \multicolumn{2}{|c|}{ BAI 1} & \multicolumn{2}{|c|}{ BAI 2} & \multicolumn{2}{|c|}{ BAI 1} & \multicolumn{2}{|c|}{$\mathrm{BAI} 2$} & \\
\hline & $M$ & DP & M & DP & M & DP & M & DP & \\
\hline \multirow[t]{2}{*}{ Beck (BAl) } & 12,9 & 7,92 & 7,9 & 5,89 & 11,3 & 7,07 & 8,2 & 8,46 & $\begin{array}{l}\text { BAl 1-GC } \times \text { GE: } p=0,34 \\
\text { BAl } 2 \text { - GC } \times \text { GE: } p=0,46 \\
\text { Intragrupo } \\
\text { teste U de Mann-Whitney } \\
\text { GE - BAl } 1 \times \text { BAl } 2: p=0,0323^{*} \\
\text { GC - BAl } 1 \times \text { BAl } 2: p=0,14\end{array}$ \\
\hline & \multicolumn{2}{|c|}{ M } & \multicolumn{2}{|c|}{ DP } & \multicolumn{2}{|c|}{ M } & \multicolumn{2}{|c|}{ DP } & \\
\hline $\begin{array}{l}\text { Exame Laboratorial de } \\
\text { Cortisol }\end{array}$ & \multicolumn{2}{|c|}{$\begin{array}{l}\text { manhã: } 5,75 \\
\text { tarde: } 9,06\end{array}$} & \multicolumn{2}{|c|}{$\begin{array}{l}\text { manhã: 4,03 } \\
\text { tarde: 3,59 }\end{array}$} & \multicolumn{2}{|c|}{$\begin{array}{l}\text { manhã: 15,36 } \\
\text { tarde: } 13\end{array}$} & \multicolumn{2}{|c|}{$\begin{array}{c}\text { manhã: } 11,03 \\
\text { tarde: } 5,35\end{array}$} & $\begin{array}{l}\text { teste t de Student } \\
p=0,031^{*}\end{array}$ \\
\hline $\begin{array}{l}\text { Comportamento ao } \\
\text { despertar da anestesia } \\
\text { (UTI) }\end{array}$ & \multicolumn{2}{|c|}{$M=12,9$} & \multicolumn{2}{|c|}{$\mathrm{DP}=1,97$} & \multicolumn{2}{|c|}{$M=9,6$} & \multicolumn{2}{|c|}{$\mathrm{DP}=2,98$} & $\begin{array}{l}\text { teste t de Student } \\
p=0,0031^{*}\end{array}$ \\
\hline Evolução Clínica & \multicolumn{2}{|c|}{$M=9,15$} & \multicolumn{2}{|c|}{$\mathrm{DP}=1,29$} & \multicolumn{2}{|c|}{$M=8,46$} & \multicolumn{2}{|c|}{$\mathrm{DP}=1,45$} & $\begin{array}{l}\text { teste t de Student } \\
p=0,017^{*}\end{array}$ \\
\hline
\end{tabular}

M: média; DP: Desvio-padrão; UTI: unidade de terapia intensiva; GC: grupo-controle; GE: grupo-experimental. 
corporal e a pressão arterial em níveis desejáveis, repercutindo em melhor evolução clínica.

O resumo dos resultados obtidos com cada instrumento e a respectiva análise estatística está apresentado na Tabela 5.

\section{Considerações Finais}

Os resultados deste estudo indicaram efeitos benéficos prováveis da intervenção tanto no nível de stress e de ansiedade, como no comportamento do paciente no período pós-cirúrgico. É possível que a intervenção tenha afetado a evolução clínica diretamente por meio da mudança de probabilidade de comportamentos relevantes, e através de influências no modo de enfrentamento das situações estressoras do ambiente hospitalar.

Embora os resultados apontem para a possibilidade de ter sido efetiva a intervenção realizada, a amostra utilizada foi bastante limitada numericamente. Características de ambiente físico, social e do tratamento, apesar de semelhantes à maioria dos hospitais gerais do país, foram as mesmas para todos os participantes, o que pode dificultar comparações com amostras de perfil sociodemográfico diferentes. Generalizações dos resultados também ficam restritas.

Outra limitação do estudo está no fato de ter sido a avaliação do comportamento do paciente na UTI realizada por apenas um observador, sem outro registro objetivo como a filmagem, por exemplo. Há também o fato de se ter coletado apenas uma amostra de cortisol, o que pode ter limitado o alcance da interpretação dos resultados. Não foi aplicado qualquer inventário para avaliar o stress, e essa medida teria sido útil para complementar os dados obtidos com o exame de cortisol.

Sugere-se que sejam conduzidas pesquisas com maior número de sujeitos e com maior controle do registro dos operantes na UTI. Seria interessante também que pesquisas posteriores utilizassem o Inventário de Sintomas de Stress de Lipp (Lipp, 2000) - por ser esse o instrumento mais utilizado hoje no país para averiguar a fase e o tipo de stress (agudo ou crônico) dos participantes - e duas medidas de cortisol salivar (pré-teste e pós-teste), evitando a introdução de mais um procedimento aversivo para o paciente.

\section{Referências}

Amaral, V. L. A. R. (2001). Análise contingencial como um modelo de intervenção breve em psicologia da saúde. In M. L. Marinho \& V. E. Caballo (Orgs.), Psicologia clínica e da saúde (pp.279-294). Londrina: UEL.

Amaral, V. L. A. R., \& Albuquerque, S. R. T. P. (2000). Crianças com problemas crônicos de saúde. In E. F. M. Silvares (Org.), Estudos de caso em psicologia clínica comportamental infantil (pp.219-232). Campinas: Papirus.

Beck, A. T., Brown, G., Epstein, N., \& Steer, R. A. (1988). An inventory for measuring clinical anxiety. Journal of Consulting and Clinical Psychology, 56, 893-897.

Broering, C. V., \& Crepaldi, M. A. (2008). Preparação psicológica para a cirurgia em Pediatria: Importância, técnicas e limitações. Paidéia, 18 (39), 61-72.

Catania, A. C. (1999). Aprendizagem: comportamento, linguagem e cognição. Porto Alegre: Artmed.

Costa Jr., A. L. (2001). O desenvolvimento da psiconcologia: implicações para a pesquisa e intervenção profissional em saúde. Psicologia: Ciência e Profissão, 21 (2), 36-43.

Costa Jr., A. L. (2005). Psicologia da saúde e desenvolvimento humano: o estudo do enfrentamento em crianças com câncer e expostas a procedimentos médicos e invasivos. In M. A. Dessen, A. L. Costa Júnior (Orgs.), A ciência do desenvolvimento humano: tendências atuais e perspectivas futuras. Porto Alegre: Artmed.

Cunha, J. A. (1999). Estudos dos pontos de corte do BDI e BAI na versão em português. Pôster apresentado no $8^{\circ}$ Congresso Nacional de Avaliação Psicológica, Porto Alegre.

Galimbert, D., Ruiz, S. O., Mendes, J. A., Camargos, A. S., Cartes, R. M., \&Torres, L. A. (2007). Análises e recomendações para melhorar a adesão ao uso dos anticoncepcionais hormonais orais na América Latina. Recuperado em 2007, 28 de junho, disponível em <www.boa saude.uol.com.br/libs.

Garbossa, A., Maldaner, E., Mortari, D. M., Biasi, J., \& Leguisamo, C. P. (2009). Efeitos de orientações fisioterapêuticas sobre a ansiedade de pacientes submetidos à cirurgia de revascularização miocárdica. Revista Brasileira de Cirurgia Cardiovascular, 24 (3), 359-366.

Gorayeb, R. (2001). A prática da psicologia hospitalar. In M. L. Marinho \& V. E. Caballo (Orgs.), Psicologia clínica e da saúde (pp.263-278). Londrina: EDUEL.

Haydu, V. B. (2009). Compreendendo os processos de interação do homem com seu meio ambiente. In S. R. Souza \&V. B. Haydu (Orgs.), Psicologia comportamental aplicada: avaliação e intervenção nas áreas do esporte, clínica, saúdee educação (p.935). Londrina: EDUEL.

Kain, Z. N., Caldwell-Andrews, A. A., Mayes, L. C., Weinberg, M. E., Wang, S. M., MacLaren, J. E, et al. (2007). Familycentered preparation for surgery improves perioperative outcomes in children: a randomized controlled trial. Anesthesiology, 106 (1), 65-74.

Lipp, M. E. N. (2000). Inventário de sintomas de stress de Lipp. São Paulo: Casa do Psicólogo. 
Lipp, M. E. N., Frare, A., \& Santos, F. U. (2007). Efeitos de variáveis psicológicas na reatividade cardiovascular em momentos de stress emocional. Estudos de Psicologia (Campinas) 24 (2), 161-167. doi: 10.1590/S0103-166X200700 0200003.

Malerbi, F. E. K. (2001). Estratégias para aumentar a adesão em pacientes com diabetes. In H. Guilhardi, M. B. B. P. Nadi, P. P. Queiroz \& M. C. Scoz (Orgs.), Sobre comportamento e cognição: expondo a variabilidade (Vol.7, pp.126-131). Santo André: ESETec Editora.

Manfro, G. G, Alexandre Neto, C., Pollack, M., Mezzomo, K. M., Preffer, F. (2003). Stress regulates the lymphocyte homing receptor. Arquivo de Neuropsiquiatria, 61 (1), 20-24.

Marcolino, J. A. M., Suzuki, F. M., Alli, L. A. C., Gozzani, J. L., \& Mathias, L. A. S. T. (2007). Medida da ansiedade e da depressão em pacientes no pré-operatório: estudo comparativo. Revista Brasileira de Anestesiologia, 57 (2), 157-166.

Miyazaki, M. C. O. S., Domingos, N. A. M., \& Caballo, V. E. (2001). Psicologia da saúde: intervenções em hospitais públicos. In B. Range (Org.), Psicoterapias cognitivo-comportamentais (pp.463-474). Porto Alegre: Artmed.

Pires, M. T. B. (2001). Revascularização cirúrgica do miocárdio. Belo Horizonte: Edump.

Santana J. J. R. A., Fernandes, L. F. B., Zanin, C. R., Waeteman, C. M., \& Soares, M. (2010). Grupo educativo de cirurgia cardíaca em um hospital universitário: impacto psicológico. Estudos de Psicologia (Campinas), 27 (1), 31-39. doi: 10.1590/S0103-166X2010000100004.

Shimidt, A., \& Souza, D. G. (2003). Controle instrucional: discussões atuais. In C. E. Costa, J. C. Luzia \& H. H. N. Sant'Anna (Orgs.), Primeiros passos em análise do comportamento e cognição (pp.33-54). Santo André: ESETec Editores Associados.
Silva Jr., S. C. (2000). Fisiologia e manejo da dor. In R. R. Kerbauy (Org.), Sobre comportamento e cognição: conceitos, pesquisa e aplicação, a ênfase no ensinar, na emoção e no questionamento clínico (pp.133-138). São Paulo: ESETec Editores.

Smith, T. (1996). Endorfinas. Enciclopédia Prática de Medicina, $6(1), 434$.

Sousa, L. M., \& Souza Filho, E. A. (2008). Percepções sociais de pacientes sobre profissionais de saúde e outros estressores no ambiente de unidade de terapia intensiva. Estudos de Psicologia (Campinas), 25 (3), 333-342. doi: 10.1590/S0103-166X2008000300002.

Umann, J., Guido, L. A., \& Linch, G. F. C (2010). Estratégias de enfrentamento a cirurgia cardíaca. Ciência, Cuidado e Saúde, $9(1), 67-73$.

Valgas, M. A. (2004). Efeitos do controle instrucional sobre o comportamento. Dissertação de mestrado não-publicada, Pontifícia Universidade Católica de São Paulo.

Werlang, S. C., Azzolin, K., Moraes, M. A., \& Souza, E. N. (2008). Comunicação não verbal do paciente submetido à cirurgia cardíaca: do acordar da anestesia à extubação. Revista Gaúcha de Enfermagem, 29 (4), 551-556.

Zakir, N. S. (2010). Influências parentais nas interações do stress com criatividade e com tempo livre em adolescentes. Tese de doutorado não-publicada, Programa de Pós-Graduação em Educação, Universidade Federal do Paraná, Curitiba.

Recebido em: 29/4/2010

Versão final reapresentada em: 10/1/2011

Aprovado em: 12/1/2011 
